OPEN ACCESS

Edited by:

Natalie Julie Serkova,

University of Colorado,

United States

Reviewed by:

S. Gene Kim,

Cornell University, United States

Subathra Adithan,

Jawaharlal Institute of Postgraduate

Medical Education and Research

(JIPMER), India

${ }^{*}$ Correspondence:

Xiao-Ping Yin

browngao@126.com;

yinxiaoping78@sina.com orcid.org/0000-0002-7386-1069

Specialty section:

This article was submitted to

Cancer Imaging and

Image-directed Interventions,

a section of the journal

Frontiers in Oncology

Received: 28 January 2021 Accepted: 28 April 2021

Published: 26 May 2021

Citation:

Pei $X$, Wang $P$, Ren J-L, Yin $X-P$, Ma $L-Y$, Wang $Y$, Ma $X$ and Gao $B-L$

(2021) Comparison of Different Machine Models Based on ContrastEnhanced Computed Tomography Radiomic Features to Differentiate High From Low Grade Clear Cell Renal Cell Carcinomas.

Front. Oncol. 11:659969. doi: 10.3389/fonc.2021.659969

\section{Comparison of Different Machine Models Based on Contrast-Enhanced Computed Tomography Radiomic Features to Differentiate High From Low Grade Clear Cell Renal Cell Carcinomas}

\author{
Xu Pei ${ }^{1}$, Ping Wang ${ }^{1}$, Jia-Liang Ren ${ }^{2}$, Xiao-Ping Yin ${ }^{1,3 *}$, Lu-Yao Ma ${ }^{1}$, Yun Wang ${ }^{1}, X_{i} \mathrm{Ma}^{1}$ \\ and Bu-Lang Gao ${ }^{1}$ \\ ${ }^{1}$ CT/MRI Room, Affiliated Hospital of Hebei University, Baoding, China, ${ }^{2}$ Department of Pharmaceutical Diagnostics, \\ GE Healthcare China (Shanghai) Co Ltd., Shanghai, China, ${ }^{3}$ Key Laboratory of Cancer Radiotherapy and Chemotherapy \\ Mechanism and Regulations, Baoding, China
}

Purpose: This study was to investigate the role of different radiomics models with enhanced computed tomography (CT) scan in differentiating low from high grade renal clear cell carcinomas.

Materials and Methods: CT data of 190 cases with pathologically confirmed renal cell carcinomas were collected and divided into the training set and testing set according to different time periods, with 122 cases in the training set and 68 cases in the testing set. The region of interest $(\mathrm{ROI})$ was delineated layer by layer.

Results: A total of 402 radiomics features were extracted for analysis. Six of the radiomic parameters were deemed very valuable by univariate analysis, rank sum test, LASSO cross validation and correlation analysis. From these six features, multivariate logistic regression model, support vector machine (SVM), and decision tree model were established for analysis. The performance of each model was evaluated by AUC value on the ROC curve and decision curve analysis (DCA). Among the three prediction models, the SVM model showed a high predictive efficiency. The AUC values of the training set and the testing set were 0.84 and 0.83 , respectively, which were significantly higher than those of the decision tree model and the multivariate logistic regression model. The DCA revealed a better predictive performance in the SVM model that possessed the highest degree of coincidence.

Conclusion: Radiomics analysis using the SVM radiomics model has highly efficiency in discriminating high- and low-grade clear cell renal cell carcinomas.

Keywords: renal clear cell carcinoma, enhanced computed tomography, imaging histology, logistic regression, radiomics 


\section{INTRODUCTION}

Clear cell renal cell carcinoma (ccRCC) accounts for $70 \%$ of renal cancers (1). Since the long-term survival of clear cell carcinoma patients correlates negatively to the Fuhrman grading (2-4), it is crucial to accurately grade clear cell carcinoma of the kidney as early as possible. Grading ccRCC through aspiration biopsy is controversial as the operation itself carries risk of metastatic spread $(5,6)$. Previous studies on RCC were mostly based on analysis of images of conventional computed tomography (CT) (7-9), which was often interfered by human factors and lack of quantification. Through precise quantitative analysis of medical images, radiomics provides researchers an effective way to detect biological characteristic changes caused by tumor microenvironment (10-12). Classic CT information or CT-based radiomics has been applied to establish predictive models for ccRCC grade. In three logistic regression models of radiomics based on non-texture features, texture fraction and non-texture feature combined with texture fraction for identifying high- and low-grade ccRCCs (13), the area under the operating curve (AUC) values in the three models were $0.826,0.878$, and 0.843 for the training set and $0.671,0.771$, and 0.780 for the testing set, respectively. Some image features like tumor size (TS) and permeability surface-area product (PS) were helpful in differentiating high- from low-grade ccRCCs based on conventional CT studies, with the AUC of TS and PS of 0.7 (14). The sensitivity and specificity were 0.8 and 0.6 for TS and 0.7 and 0.8 for PS, respectively. Moreover, gene fragments and radiomics can be combined to establish a two-group model for differentiating ccRCC from non-clear cell RCC (non-ccRCC), with the AUC of the training set and testing set being 0.969 and 0.900 , respectively (15). Some studies confirmed that necrosis can independently predict the biological invasiveness of ccRCCs $(16,17)$. Moreover, only the logistic regression model was utilized in most of these studies lacking comparison between different predictive modeling methods. Therefore, in this study, three models including logistic regression, decision tree and support vector machine (SVM) were established and compared for ccRCC grading performance.

\section{MATERIALS AND METHODS}

\section{Patients}

This retrospective study was approved by the Ethics Review Committee of Affiliated Hospital of Hebei University with all patients given their signed informed consent. All methods were performed in accordance with the relevant guidelines and regulations. Patients with ccRCC were enrolled between January, 2017 and December, 2018 in our hospital. Inclusion criteria were a single lesion with clear grades of RCC and preoperative enhanced CT images in the cortical phase with fast-in and fast-out enhancement (cortical phase showed the clearest). Exclusion criteria were: (I) carcinomar metastasis, (II) cystic changes in the lesion of carcinoma, (III) necrosis volume $>80 \%$ of the maximal lesion volume, and (IV) poor image quality. In accord with these criteria, 42 unqualified samples were excluded, and 190 eligible samples were included. In this study, I-II grade ccRCC was defined as low-grade renal clear cell carcinoma, and III-IV grade ccRCC was defined as high-grade renal carcinoma (18) (Figure 1). Among the qualified 190 patients with ccRCC, 133 cases were of grade I-II ccRCC and 57 cases were of grade III-IV ccRCC, including 98 males and 92 females with an age range of $27-88$ years (mean $58.30 \pm 8.70$ ) (Table 1). Their maximal diameters of the carcinoma ranged 2$12 \mathrm{~cm}$ (mean $5.6 \pm 4.4$ ) from post-operative pathological exams.

\section{CT Image Acquisition}

Abdominal plain and enhancement CT scans were performed with a 64-row CT scanner (GE Discovery HD 750, GE Health Care, Chicago, IL, USA). Contrast agent was iodophor alcohol, a non-ionic iodine contrast agent. The post-injection scanning time points were $30-35 \mathrm{~s}, 50-60 \mathrm{~s}$ and $180 \mathrm{~s}$, covering the medullary phase and renal pelvis stage. Scanning parameters were as follows: cortical phase, pitch: $0.984: 1$, layer thickness: $5 \mathrm{~mm}$, field of view: $40 \mathrm{~cm} \times 40 \mathrm{~cm}$, matrix: $512 \times 512$, tube voltage: 100-120 kV, tube current: 134-409 mA, window width: 250-450 $\mathrm{HU}$, and window position: $30-50 \mathrm{HU}$.

\section{Volumes of Interest (VOIs) Segmentation}

The cortical phase images of enhanced CT from 190 subjects were imported into the ITK-SNAP software (19), and the region of interest (ROI) was delineated by one radiologist with 8 years of working experience and checked by another radiologist with 10 years of working experience.

\section{Radiomics Feature Extraction and Selection}

The radiomics features were extracted from the original and filtered images with the AK software (Artificial Intelligence Kit V3.0.0.R, GE Healthcare, China). A total of 402 features were obtained, including 42 histogram features, 144 gray-level cooccurrence matrices features (GLCM), 180 gray-level run length matrices features (GLRM), 11 gray-level zone matrices features (GLSZM), 15 shape-based features, and 10 Haralick features. The feature selection procedure was as follows: Firstly, the data of patients from January 2017 to April 2018 were included in the training set, and the data of patients from April 2018 to December 2018 were included in the testing set, with the data of 122 patients in the training set (with 81 cases of I-II ccRCC and 41 cases of IIIIV ccRCC) and 68 patients in the testing set (with 52 cases of I-II ccRCC and 16 cases of III-IV ccRCC). Secondly, the data were preprocessed, including replacing missing values with the median value and standardizing the Z-score of features in all data. Thirdly, the extracted features were analyzed by one-way ANOVA and Wilcox rank-sum test, with the significant $P$ value set at less than 0.05 . Then, the least absolute shrinkage and selection operator (LASSO) method, which has been shown to be suitable for high dimensional data analysis (13), were used for further feature screening. The LASSO method selects features using a tuning parameter (Lambda), with some coefficients in the covariance can be shrunk to zero when the cross-validation error 

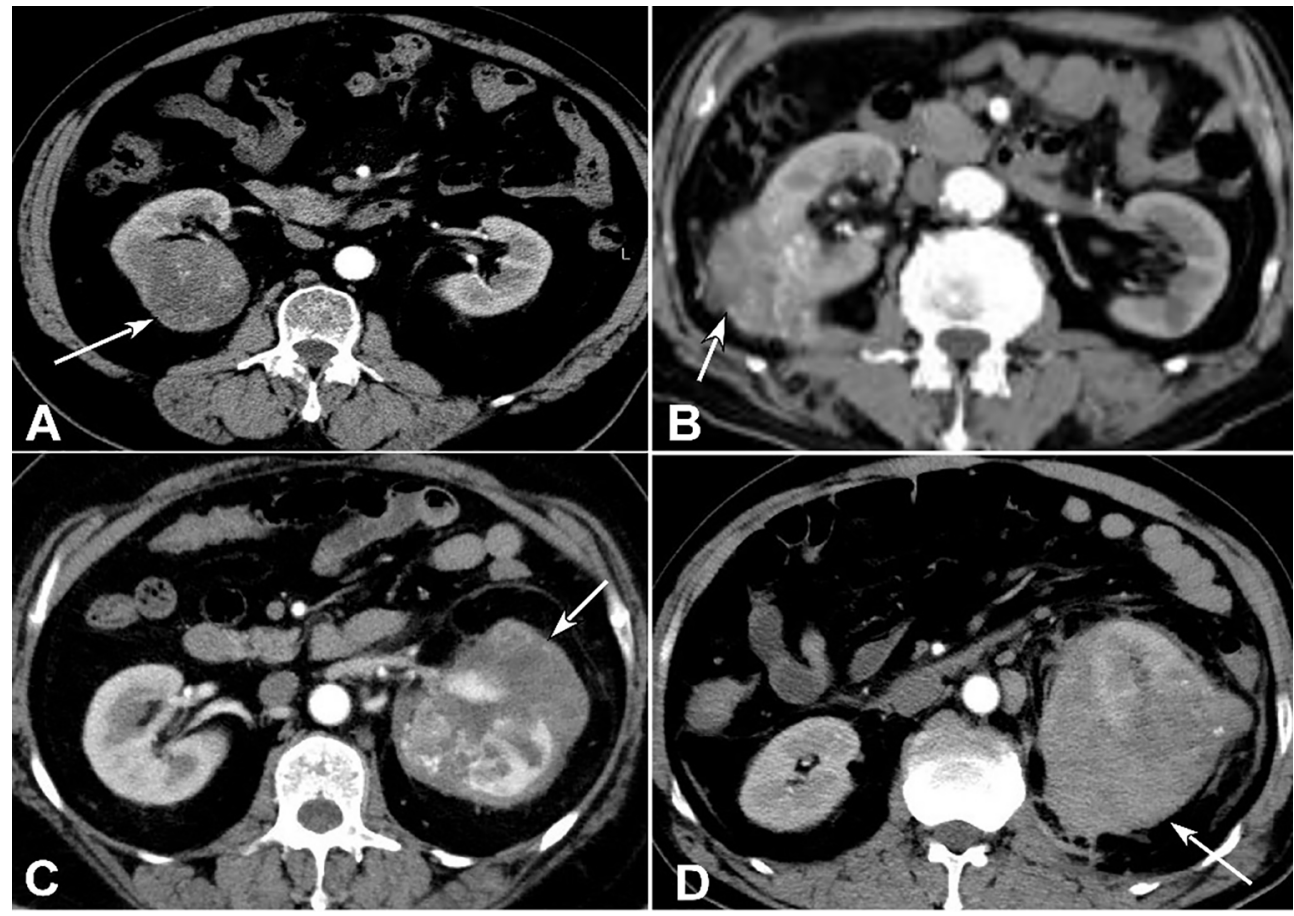

FIGURE 1 | Clear cell renal cell carcinomas (ccRCC) with different grades. (A) Grade I ccRCC (arrow) was demonstrated. (B) Grade II ccRCC was shown (arrow). (C) Grade III ccRCC was revealed (arrow). (D) Grade IV ccRCC (arrow) was displayed.

is the smallest. All the feature selection procedure performed on the training set and applied on the testing set. The finally selected features were used to construct models.

\section{RADIOMIC MODEL BUILDING AND VALIDATION}

The ROC curves of each model in the training set (data of 122 patients) and testing set (68 patients) were calculated with all available patients and the AUC values were derived (Figure 3).

TABLE 1 | Demography of patients in two sets.

\begin{tabular}{lcc}
\hline Variables & Training set & Testing set \\
\hline Case no. & $122(64 \%)$ & $68(36 \%)$ \\
Sex & & \\
$\quad$ Male & $66(54 \%)$ & $33(49 \%)$ \\
$\quad$ Female & $56(46 \%)$ & $35(51 \%)$ \\
Age mean (range, y) & $55.6(28-85)$ & $56.1(31-87)$ \\
$\quad<60$ & $59(48 \%)$ & $35(51 \%)$ \\
$\quad 60$ & $33(49 \%)$ & $63(52 \%)$ \\
Subtype & & \\
$\quad$ Low-grade ccRCC & $81(66 \%)$ & $52(76 \%)$ \\
$\quad$ High-grade ccRCC & $41(34 \%)$ & $16(24 \%)$ \\
Tumor size (cm, mean \pm SD) & & \\
$\quad$ Low-grade ccRCC & $6.48 \pm 3.46$ & $6.57 \pm 3.31$ \\
$\quad$ High-grade ccRCC & $7.21 \pm 3.13$ & $8.31 \pm 3.31$ \\
\hline
\end{tabular}

Low grade, grades I-II; High-grade, grades III-IV; SD, standard deviation.
The predictive performances of three models (logistic regression, decision tree, and SVM) were compared for analysis. The decision curve analysis (DCA) was conducted to evaluate the clinical usefulness of the models for ccRCC prediction. DCA quantified the net benefits at different threshold probabilities in the training and testing set (Figure 4).

\section{Statistical Analysis}

Statistical analysis was performed with the R software (version: 3.6.3, www.r-project.org). The Chi-square test was used to evaluate the distribution difference in high and low-grade cc RCCs. The LASSO, SVM, and decision tree model were conducted based on 'glmnet', 'e1071', and 'rpart' packages, respectively. The receiver operating characteristics (ROC) curve analysis was performed to determine the AUC, accuracy, specificity and sensitivity for evaluating the performance of the model. The significance was set at $P<0.05$.

\section{RESULTS}

The six most valuable features selected by LASSO for radiomics modelling were GLCMEntropy, GreyLevelNonuniformity, ShortRunEmphasis, LongRunLowGreyLevelEmphasis, ShortRunLowGreyLevelEmphasis, and IntensityVariability. The LASSO regression was shown in Figure 2. The specific parameters and feature extraction used in the six most valuable features were demonstrated in Table 2. These features were used 
to establish three models of logistic regression, decision tree and SVM in the training set with 122 patients. Each model was trained and assessed using the repeated ten-fold cross-validation method in the training set. Performance of differentiating high grade from low grade ccRCC was evaluated with the testing set (68 patients) (Figure 3).

The AUC values in the training set and testing sets are respectively 0.63 (95\% CI $0.53-0.73$ ) and 0.64 (95\% CI $0.48-0.8)$ with the logistic regression model, 0.84 (95\% CI 0.76-0.92) and 0.83 (95\% CI 0.69-0.96) with SVM model, and finally, 0.69 (95\% CI $0.60-0.78)$ and 0.72 (95\% CI 0.56-0.87) with the decision tree model. The cutoff value of each model was obtained from the Youden index from the ROC curve, with the value being 0.366 , 0.38 , and 0.276 , respectively, in the logistic regression, SVM, and decision tree for the test set. The results presented in Tables $\mathbf{3}$ and $\mathbf{4}$ showed that the SVM model had achieved the best performance.

DCA was conducted to evaluate clinical usefulness of the models in prediction by quantifying the net benefits (relative benefits), at different threshold probabilities in both sets (Figure 4). The SVM model had the best performance in prediction of low- and high- grade renal cell carcinoma. In the DCA analysis (Figure 4), the SVM model was shown to obtain the highest benefit in the range of 0.34-0.49 which contained the cutoff value 0.38 for the SVM model. The "benefit" was relative and indicated the efficiency of the models in the test set.

The prediction performance of the three models for low and high grade RCC was verified and compared (Figures 5-7). There was no significant $(P=0.054)$ difference in the high and low-grade distribution of ccRCCs between the training and testing sets.

In verification of the logistic regression model (Figure 5), the true negative rate (specificity) for predicting grade I-II ccRCC was $60.5 \%(49 / 81)$ in the training and $53.7 \%(22 / 41)$ in the testing set, and the true positive rate (sensitivity) for predicting grade III-IV ccRCC was $65.4 \%$ (34/52) in the training and $75 \%$ $(12 / 16)$ in the testing set. In verification of the SVM model (Figure 6), the true negative rate (specificity) was $76.5 \%(62 / 81)$ for predicting grade I-II ccRCC in the training and $85.4 \%(35 / 41)$ in the testing set, and the true positive rate (sensitivity) was $84.6 \%(44 / 52)$ in the training set. The testing set also exhibited a true negative rate of $75 \%(12 / 16)$ for predicting grade III-IV ccRCC. In verification of the decision tree model (Figure 7), the true negative rate (specificity) was $77.8 \%$ (63/81) for predicting grade I-II ccRCC with the true positive rate (sensitivity) of $55.8 \%$ $(29 / 52)$ in the training set. For the testing set, the true negative rate was $82.9 \%(34 / 41)$ for predicting grade I-II ccRCC and $62.5 \%(10 / 16)$ for predicting grade III-IV ccRCC.
A

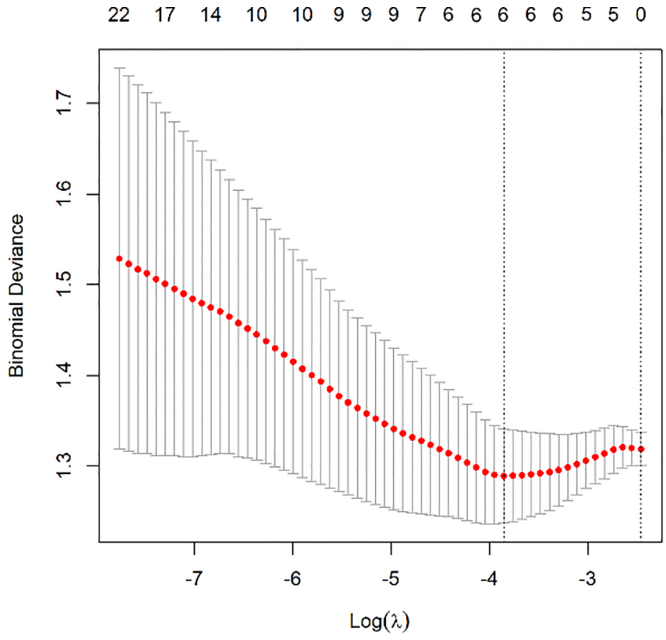

B

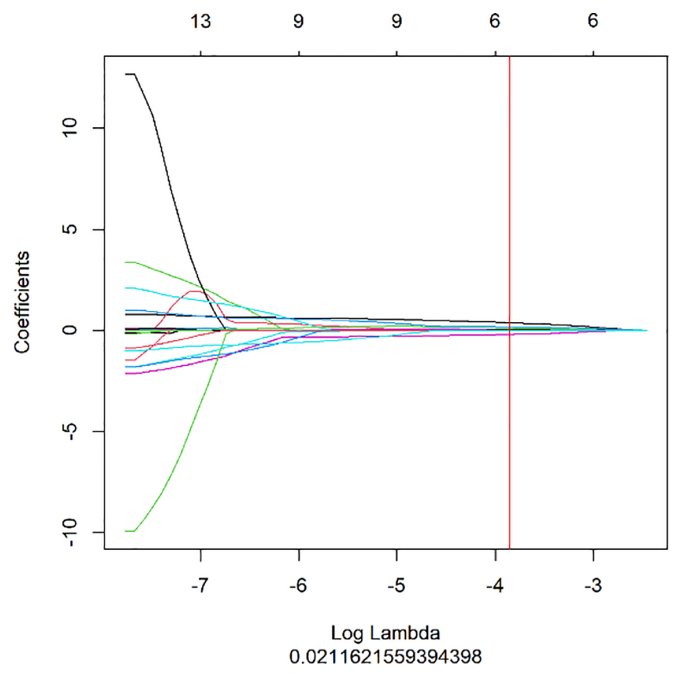

FIGURE 2 | Feature selection with the LASSO method. (A) The tuning parameter ( $\lambda$ ) changes in the LASSO model. The binomial deviance curve was generated with the $\log (\lambda)$. The minimum criteria for five-fold cross-validation were applied. The best $\lambda=0.0212$ was obtained at the minimal binomial deviance. (B) The LASSO coefficient profile plot with different $\log (\lambda)$ was shown. The vertical red line was the best $\lambda$ with 6 selected radiomic features.

TABLE 2 | Specific parameters and feature extraction in six features.

\begin{tabular}{|c|c|c|c|c|}
\hline ID & Class & Type & Offset & Direction \\
\hline 2 & RLM & GreyLevelNonuniformity & 7 & All (3D) \\
\hline 5 & RLM & ShortRunLowGreyLevelEmphasis & 4 & All (3D) \\
\hline 6 & Histogram & IntensityVariability & - & - \\
\hline
\end{tabular}



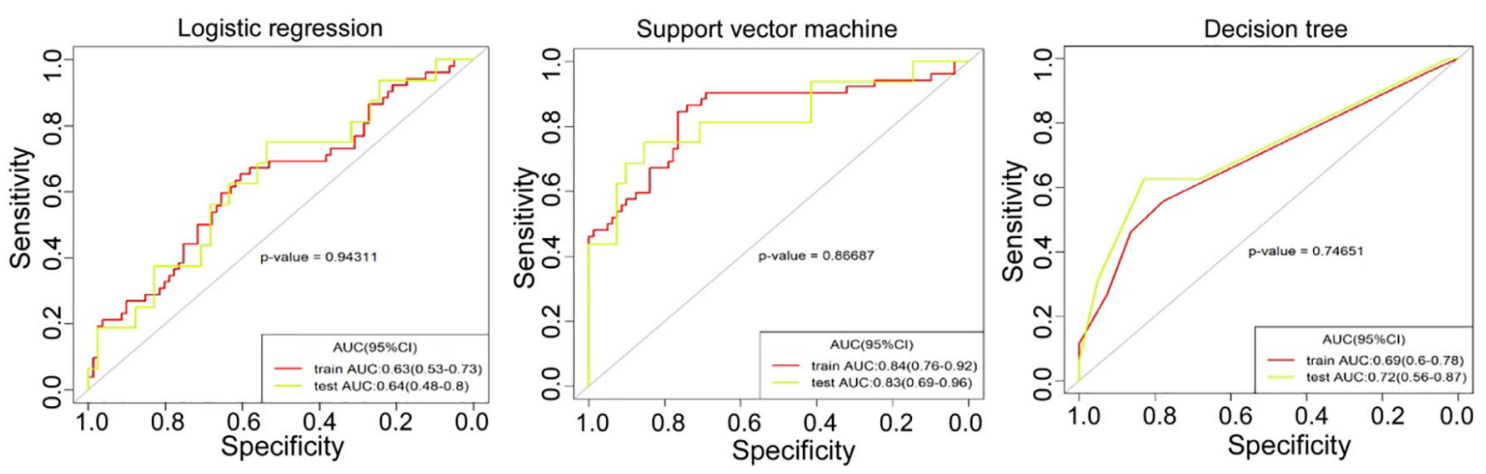

FIGURE 3 | The receiver operating characteristics (ROC) curve analysis was performed for three models of logistic regression, support vector machine and decision tree in the training set and testing set.

The AUC, accuracy, specificity, and sensitivity were used to evaluate the performance of the model (Tables 1 and 2). Through comprehensive comparison of the AUC value, specificity, sensitivity of the three models, the best prediction efficiency, observed in the sSVM model, was therefore selected for prediction purpose in this study. The SVM model had the greatest accuracy (0.797 and 0.825$)$, sensitivity (0.846 and 0.825$)$ and specificity ( 0.742 and 0.750$)$ in both the training and testing set compared with the logistic regression model ( 0.624 and 0.596 , 0.654 and $0.750,0.605$ and 0.537 , respectively) and the decision tree model (0.692 and $0.772,0.558$ and $0.625,0.778$ and 0.829 , respectively).

\section{DISCUSSION}

The present study was aimed at the differentiation of high- from low-grade ccRCCs, because pathological grades highly correlate with ccRCC metastasis and prognosis (20). ccRCC has different clinical prognoses at different grades, and early identification of pathological grade of ccRCCs is valuable for timely clinical treatment and patient health.

Radiomics analysis is to extract a multitude of features form medical images to analyze size, shape, and texture, with useful spatial information on pixel or voxel distribution and modes. The recent advancements in the study of ccRCCs were based on imaging histology except for its grading (21). In the modeling and identification of high- and low-grade ccRCCs, previous studies $(22,23)$ used in vivo diffusion-weighted imaging (DWI) and imaging histology to achieve the AUC value of 0.8 , whereas an AUC value of 0.73 was reached by the Renometric score based on CT imaging in identification of high-level RCCs (23). The AUC values for SVM model in the training and testing sets in our study were 0.84 and 0.83 , respectively, higher than 0.8 or 0.73 of methods described earlier.

Ding et al. (13) applied radiomics to establish three logistic regression models to identify high and low-grade ccRCCs, achieving the AUC values in the training sets of the three

TABLE 3 | ROC curve analysis of three models in the training set.

\begin{tabular}{lccc}
\hline Parameter & Logistic (Train) & SVM (Train) & Decision Tree (Train) \\
\hline AUC & $0.632(\mathrm{Cl}: 0.533-0.730)$ & $0.840(\mathrm{Cl}: 0.653-0.758)$ & $0.688(\mathrm{Cl}: 0.601-0.775)$ \\
Accuracy & $0.624(\mathrm{Cl}: 0.530-0.707)$ & $0.797(\mathrm{Cl}: 0.719-0.862)$ & $0.692(\mathrm{Cl}: 0.606-0.769)$ \\
Sensitivity & $0.654(\mathrm{Cl}: 0.462-0.788)$ & $0.846(\mathrm{Cl}: 0.558-0.942)$ & $0.558(\mathrm{Cl}: 0.385-0.681)$ \\
Specificity & $0.605(\mathrm{Cl}: 0.272-0.741)$ & $0.742(\mathrm{Cl}: 0.284-0.852)$ & $0.778(\mathrm{Cl}: 0.575-0.904)$
\end{tabular}

ROC, Receiver operating characteristic; AUC, area under the operating curve; $\mathrm{Cl}$, confidence interval.

TABLE 4 | ROC curve analysis of three models in the testing set.

\begin{tabular}{lccr}
\hline Parameter & Logistic regression & Support vector machine & Decision Tree \\
\hline AUC & $0.639(\mathrm{Cl}: 0.476-0.802)$ & 0.826 (Cl: $0.688-0.964)$ & $0.717(\mathrm{Cl}: 0.564-0.871)$ \\
Accuracy & $0.596(\mathrm{Cl}: 0.458-0.724)$ & 0.825 (Cl: $0.701-0.913)$ & $0.772(\mathrm{Cl}: 0.642-0.873)$ \\
Sensitivity & $0.750(\mathrm{Cl}: 0.436-0.938)$ & $0.750(\mathrm{Cl}: 0.438-0.938)$ & $0.625(\mathrm{Cl}: 0.320-0.812)$ \\
Specificity & $0.537(\mathrm{Cl}: 0.195-0.756)$ & $0.854(\mathrm{Cl}: 0.341-0.976)$ & $0.829(\mathrm{Cl}: 0.400-0.951)$
\end{tabular}

ROC, Receiver operating characteristic; AUC, area under the operating curve; $\mathrm{Cl}$, confidence interval. 

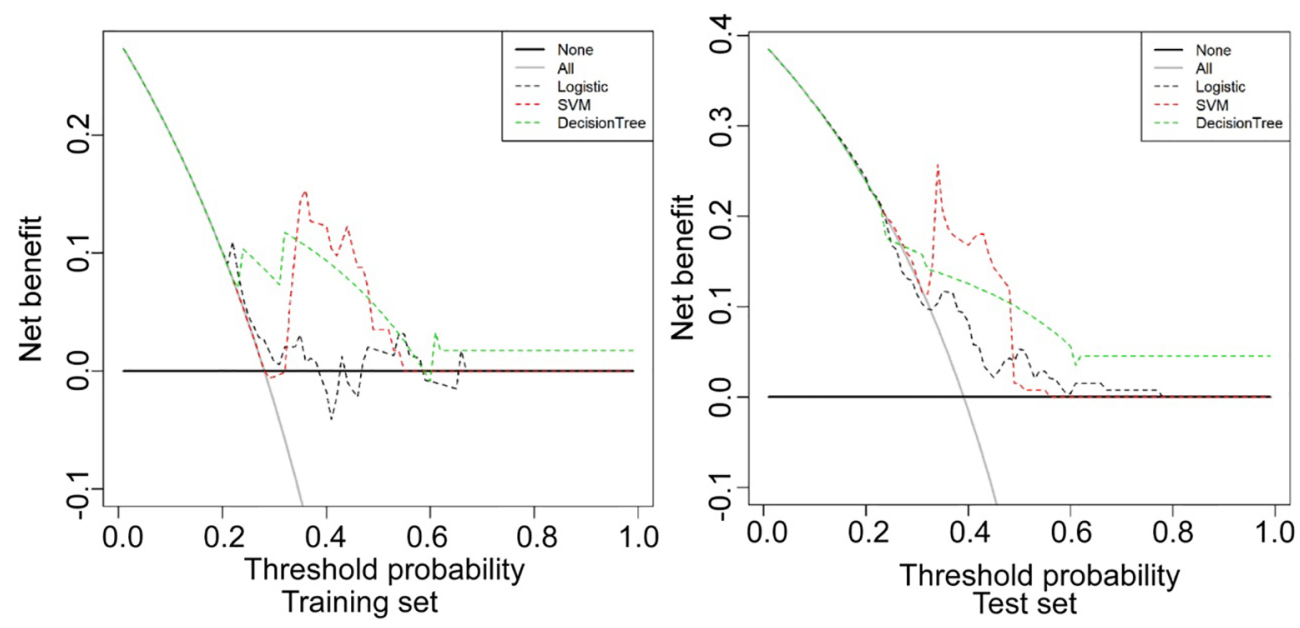

FIGURE 4 | Decision curve analysis (DCA) was conducted to evaluate the clinical usefulness of the models in prediction by quantifying the net benefits at different threshold probabilities in the training and testing set. The SVM model had the best performance in prediction of low- and high-grade renal cell carcinoma. Logistic, logistic regression model; SVM, support vector machine model; Decision tree, decision tree model.

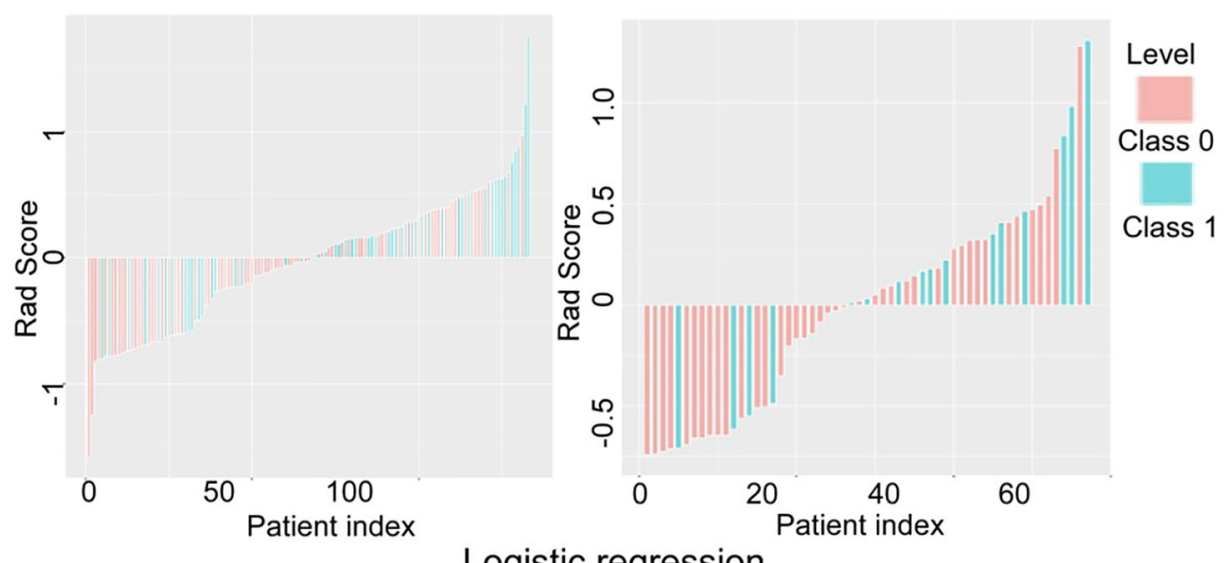

FIGURE 5 | Verification and comparison of the logistic regression model in predicting low and high grade renal cell carcinoma in the training and testing set. In the training set, the true negative rate (specificity) for predicting grade I-II clear cell renal cell carcinoma (ccRCC) was 60.5\% (49/81), and the true positive rate (sensitivity) for predicting grade III-IV ccRCC was 65.4\% (34/52). In the testing set, the true negative rate was 53.7\% (22/41) for predicting grade I-II ccRCC and 75\% (12/16) for predicting grade III-IV.

models of $0.826,0.878,0.843$ compared with the AUC values in the testing sets of $0.671,0.771$ and 0.780 , respectively. Although the results in training set were better, the scores in testing set were not as satisfactory probably due to a trend of over-fitting. In addition, Ding et al. extracted the texture features from the maximal diameter level of the mass and collected less heterogeneous information of the mass (13). Compared with the study by Ding et al, our SVM-based model performed better, with our SVM-based AUC in the training and testing set being 0.84 and 0.83 , respectively. Shu et al. (24) established three radiomic models based on renal CT enhancement images in the cortical and parenchymal phases, including cortical phase model, parenchymal phase model, and in combination. The corresponding accuracy, AUC value, sensitivity and specificity were $0.719,0.766,0.818$ and 0.822 ) for the cortical phase model, $0.738,0.602,0.693$ and 0.677 for the parenchymal phase model, and $0.777,0.838,0.838$ and 0.839 for the combined model. Comparing these results to the study with $3 \mathrm{D}$ texture analysis based model by Shu et al. (24), our results have better accuracy, AUC value, and sensitivity. Although the model produced by Shu et al. (24) possessed slightly higher specificity with the combined multi-period model outperforming the oneperiod model, their study used full data to build the model without using independent test data to validate their results. 


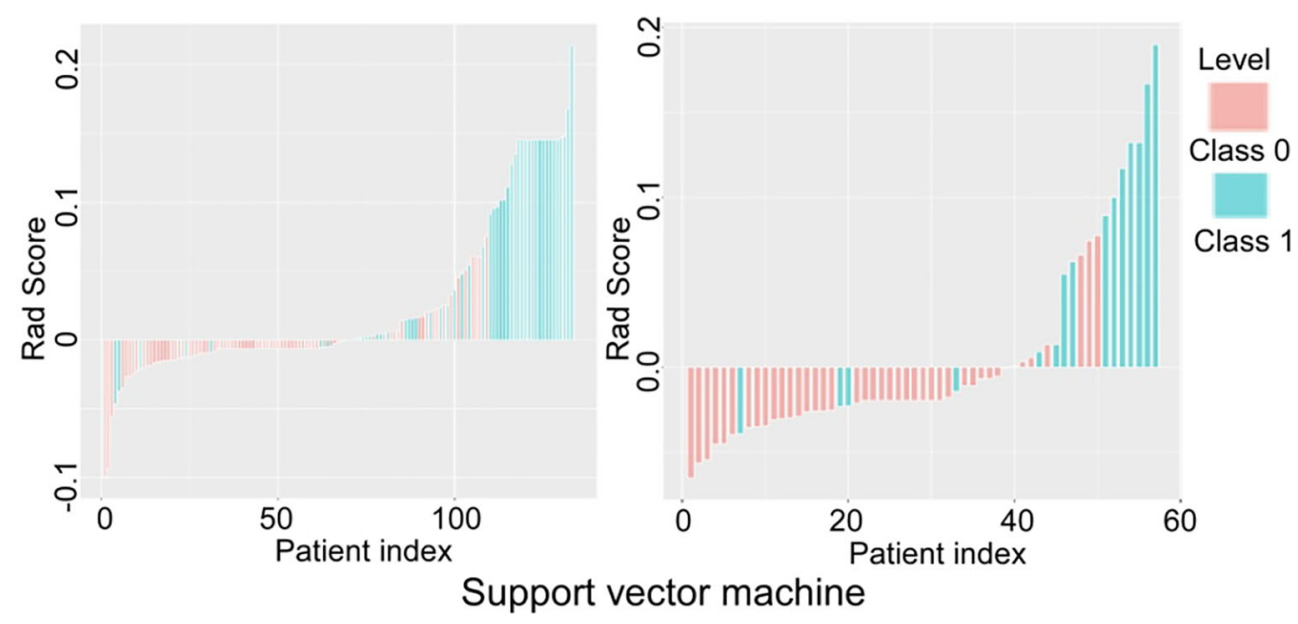

FIGURE 6 | Verification and comparison of the support vector machine model in predicting low and high grade renal cell carcinoma in the training and testing set. In the training set, the true negative rate (specificity) was $76.5 \%$ (62/81) for predicting grade I-II clear cell renal cell carcinoma (ccRCC), and the true positive rate (sensitivity) was $84.6 \%$ (44/52). In the testing set, the true negative rate was $85.4 \%$ (35/41) for predicting grade I-II ccRCC and 75\% (12/16) for predicting grade III-IV ccRCC.

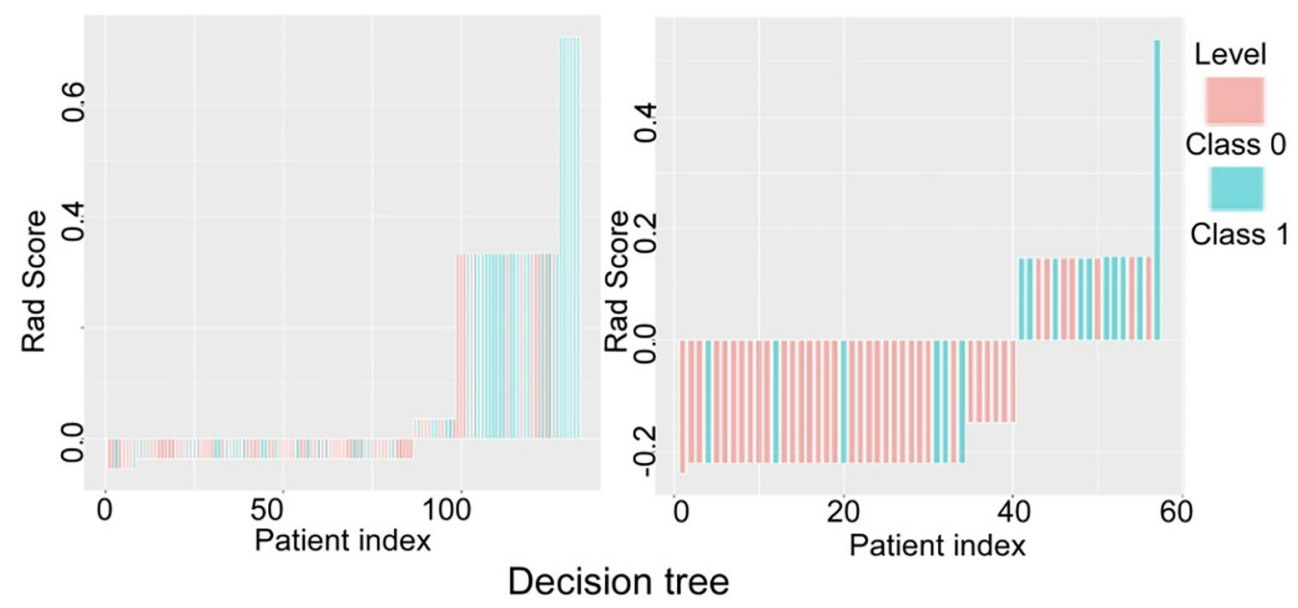

FIGURE 7 | Verification and comparison of the decision tree model in predicting low and high grade renal cell carcinoma in the training and testing set. In the training set, the true negative rate (specificity) was $77.8 \%$ (63/81) for predicting grade I-II clear cell renal cell carcinoma (ccRCC), and the true positive rate (sensitivity) was $55.8 \%$ (29/52). In the testing set, the true negative rate was $82.9 \%$ (34/41) for predicting grade I-II ccRCC and $62.5 \%$ (10/16) for predicting grade III-IV ccRCC.

Radiomics-based grading models demonstrated better performance than the model based on conventional CT parameters. Chen et al. (14) pointed out that tumor size (TS) and permeability surface-area products (PS) were helpful in distinguishing the high and low grade clear cell renal cancers, with the AUC of both TS and PS being 0.7 and the sensitivity and specificity being 0.8 and 0.6 for TS and 0.7 and 0.8 ) for PS. The grading performance in our study was also better than this study (15).

Heterogeneity is an important feature of malignant tumors and is closely related to their biological behavior. CT enhanced imaging can be used to effectively evaluate tumor heterogeneity (25). After studying low enhancement on multiphase contrastenhanced CT images for predicting presence of high tumor grade of ccRCC (26), Miles et al. found that low tumor enhancement in the cortico-medullary phases was an independent predictor of high tumor grade, which may be useful in clinical care of patients with nonsurgical approaches. It is speculated that the higher the grade of renal clear cell carcinoma, the more abundant the small capillaries (27), which is supported by another study by Li et al (15). In addition, necrosis is highly correlated with heterogeneity of tumors, which is of great significance (28). In this study (28), various processing techniques including voxel normalization and various filtering processes were used to extract a variety of high and low order features, including gray matrix and $3 \mathrm{D}$ morphological features. Finally, LASSO cross-processing was used to select the most valuable six histological features. 
After looking into a variety of common first-order features that reflect tumor heterogeneity, such as average gray level, kurtosis and entropy, Feng et al. proposed that entropy is an independent and excellent radiomic feature to describe a degree of disorder in images (29). In terms of lesion density distribution, larger entropy values suggest more randomness while smaller entropy values indicate uniformity. Thus, high-grade tumors with relatively large liquefaction necrosis volume have reduced the entropy detectable as a radiomic feature and were consequently excluded from our study. In our study, we only studied the primary renal cell carcinoma rather than metastatic carcinomas from other resources. However, if the renal cancerous lesions of the primary renal cell carcinoma contained large-area necrosis or cystic changes, they would be excluded from the study, because necrosis contained inactive tissue and cystic changes contained liquid materials. Solid mass should be retained as much as possible. The radiomics captured tissues primarily with active and biological behavior, namely solid mass tissues. Cystic degeneration and necrosis are similar in nature, and the doping of these changes in the samples may lower the evaluation efficiency of the results.

In our study, GLCM_entropy, Greylevel_Nonuniformity, and Intensity_Variability of the six features reflect the degree of random gray distribution in ROI, which is usually used to demonstrate the tumor heterogeneity. ShortRun_Emphasis and ShortRunLowGreyLevel_Emphasis are used to show the fine texture of the tumor, whereas LongRunLowGreyLevel_Emphasis is used to reflect the coarse texture within the tumor. The SVM model in our study used the RBF kernel with $\mathrm{C}$ value 1 and gamma 0.001 . The SVM is a nonlinear model which can get greater and better results than the linear model. The SVM model may be used for machine learning with small samples, for improving generalization and solving higher-dimensional problems as well as for avoiding structural selection in neural networks. There are some limitations in our study. Firstly, the overall sample size was relatively small. Secondly, patient data was not comprehensively collected, with the construction of models having excluded diagnostic elements from biochemistry, immunohistochemistry and genetic studies. Thirdly, when the VOI was delineated, the

\section{REFERENCES}

1. Znaor A, Lortet-Tieulent J, Laversanne M, Jemal A, Bray F. International Variations and Trends in Renal Cell Carcinoma Incidence and Mortality. Eur Urol (2015) 67:519-30. doi: 10.1016/j.eururo.2014.10.002

2. Ficarra V, Martignoni G, Maffei N, Brunelli M, Novara G, Zanolla L, et al. Original and Reviewed Nuclear Grading According to the Fuhrman System: A Multivariate Analysis of 388 Patients With Conventional Renal Cell Carcinoma. Cancer (2005) 103:68-75. doi: 10.1002/cncr.20749

3. Motzer RJ, Jonasch E, Agarwal N, Bhayani S, Bro WP, Chang SS, et al. Kidney Cancer, Version 2.2017, NCCN Clinical Practice Guidelines in Oncology. J Natl Compr Canc Netw (2017) 15:804-34. doi: 10.6004/jnccn.2017.0100

4. Rouprêt M, Babjuk M, Compérat E, Zigeuner R, Sylvester RJ, Cowan NC, et al. European Association of Urology Guidelines on Upper Urinary Tract Urothelial Carcinoma: 2017 Update. Eur Urol (2018) 73:111-22. doi: 10.1016/j.eururo.2017.07.036

5. Feng Z, Rong P, Cao P, Zhou Q, Zhu W, Yan Z, et al. Machine LearningBased Quantitative Texture Analysis of CT Images of Small Renal Masses: accuracy of the delineated lesions was reduced, due to unclear margins of some tumor masses or the influences by partial volume effect. Fourthly, the current single-center study lacked independent validation and evaluation from external professionals. Although our scanning parameters and reconstruction methods had been standardized, they should have been fixed with multicenter studies, thus necessitating a unified measurement standard for obtaining necessary information. Lastly, this study was limited to its retrospective nature and involvement with only Chinese ethnicity.

In summary, the current study uses radiomics analysis to differentiate the grade of ccRCC, and the support vector machine-based model exhibits the best performance for differentiating high- and low-grade ccRCC when compared to the logistic regression model and the decision tree model.

\section{DATA AVAILABILITY STATEMENT}

The raw data supporting the conclusions of this article will be made available by the authors, without undue reservation.

\section{ETHICS STATEMENT}

The studies involving human participants were reviewed and approved by Ethics committee of Affiliated Hospital of Hebei University. The patients/participants provided their written informed consent to participate in this study.

\section{AUTHOR CONTRIBUTIONS}

Study design: XP and J-LR. Data collection: XP, PW, L-YM, YW, and XM. Data analysis: XP, PW, J-LR, X-PY, and B-LG. Supervision: L-YM. Manuscript writing: XP. Revision: B-LG. All authors contributed to the article and approved the submitted version. 
Renal Cell Carcinoma. Chin Med J (Engl) (2014) 127:1640-4. doi: 10.3760/ cma.j.issn.0366-6999.20132831

10. Gillies RJ, Kinahan PE, Hricak H. Radiomics: Images are More Than Pictures, They Are Data. Radiology (2016) 278:563-77. doi: 10.1148/radiol.2015151169

11. Huang YQ, Liang CH, He L, Tian J, Liang CS, Chen X, et al. Development and Validation of a Radiomics Nomogram for Preoperative Prediction of Lymph Node Metastasis in Colorectal Cancer. J Clin Oncol (2016) 34:2157-64. doi: 10.1200/JCO.2015.65.9128

12. Fan M, Xia P, Clarke R, Wang Y, Li L. Radiogenomic Signatures Reveal Multiscale Intratumour Heterogeneity Associated With Biological Functions and Survival in Breast Cancer. Nat Commun (2020) 11:4861. doi: 10.1038/ s41467-020-18703-2

13. Ding J, Xing Z, Jiang Z, Chen J, Pan L, Qiu J, et al. CT-Based Radiomic Model Predicts High Grade of Clear Cell Renal Cell Carcinoma. Eur J Radiol (2018) 103:51-6. doi: 10.1016/j.ejrad.2018.04.013

14. Chen C, Kang Q, Xu B, Guo H, Wei Q, Wang T, et al. Differentiation of Lowand High-Grade Clear Cell Renal Cell Carcinoma: Tumor Size Versus CT Perfusion Parameters. Clin Imaging (2017) 46:14-9. doi: 10.1016/ j.clinimag.2017.06.010

15. Li ZC, Zhai G, Zhang J, Wang Z, Liu G, Wu GY, et al. Differentiation of Clear Cell and non-Clear Cell Renal Cell Carcinomas by All-Relevant Radiomics Features From Multiphase CT: A VHL Mutation Perspective. Eur Radiol (2019) 29:3996-4007. doi: 10.1007/s00330-018-5872-6

16. Hötker AM, Karlo CA, Zheng J, Moskowitz CS, Russo P, Hricak H, et al. Clear Cell Renal Cell Carcinoma: Associations Between CT Features and Patient Survival. AJR Am J Roentgenol (2016) 206:1023-30. doi: 10.2214/AJR.15.15369

17. Oh S, Sung DJ, Yang KS, Sim KC, Han NY, Park BJ, et al. Correlation of CT Imaging Features and Tumor Size With Fuhrman Grade of Clear Cell Renal Cell Carcinoma. Acta Radiol (2017) 58:376-84. doi: 10.1177/ 0284185116649795

18. Bektas S, Bahadir B, Kandemir NO, Barut F, Gul AE, Ozdamar SO. Intraobserver and Interobserver Variability of Fuhrman and Modified Fuhrman Grading Systems for Conventional Renal Cell Carcinoma. Kaohsiung J Med Sci (2009) 25:596-600. doi: 10.1016/S1607-551X(09)70562-5

19. Yushkevich PA, Piven J, Hazlett HC, Smith RG, Ho S, Gee JC, et al. UserGuided 3D Active Contour Segmentation of Anatomical Structures: Significantly Improved Efficiency and Reliability. Neuroimage (2006) 31:1116-28. doi: 10.1016/j.neuroimage.2006.01.015

20. Guðmundsson E, Hellborg H, Lundstam S, Erikson S, Ljungberg B. Metastatic Potential in Renal Cell Carcinomas $\leq 7 \mathrm{Cm}$ : Swedish Kidney Cancer Quality Register Data. Eur Urol (2011) 60:975-82. doi: 10.1016/j.eururo.2011.06.029

21. Yan L, Liu Z, Wang G, Huang Y, Liu Y, Yu Y, et al. Angiomyolipoma With Minimal Fat: Differentiation From Clear Cell Renal Cell Carcinoma and
Papillary Renal Cell Carcinoma by Texture Analysis on CT Images. Acad Radiol (2015) 22:1115-21. doi: 10.1016/j.acra.2015.04.004

22. Maruyama M, Yoshizako T, Uchida K, Araki H, Tamaki Y, Ishikawa N, et al. Comparison of Utility of Tumor Size and Apparent Diffusion Coefficient for Differentiation of Low- and High-Grade Clear-Cell Renal Cell Carcinoma. Acta Radiol (2015) 56:250-6. doi: 10.1177/0284185114523268

23. Wang HK, Zhu Y, Yao XD, Zhang SL, Dai B, Zhang HL, et al. External Validation of a Nomogram Using RENAL Nephrometry Score to Predict High Grade Renal Cell Carcinoma. J Urol (2012) 187:1555-60. doi: 10.1016/ j.juro.2011.12.099

24. Shu J, Tang Y, Cui J, Yang R, Meng X, Cai Z, et al. Clear Cell Renal Cell Carcinoma: CT-Based Radiomics Features for the Prediction of Fuhrman Grade. Eur J Radiol (2018) 109:8-12. doi: 10.1016/j.ejrad.2018.10.005

25. Ganeshan B, Miles KA. Quantifying Tumour Heterogeneity With CT. Cancer Imaging (2013) 13:140-9. doi: 10.1102/1470-7330.2013.0015

26. Miles KA, Ganeshan B, Hayball MP. CT Texture Analysis Using the Filtration-Histogram Method: What do the Measurements Mean? Cancer Imaging (2013) 13:400-6. doi: 10.1102/1470-7330.2013.9045

27. Zhu YH, Wang X, Zhang J, Chen YH, Kong W, Huang YR. Low Enhancement on Multiphase Contrast-Enhanced CT Images: An Independent Predictor of the Presence of High Tumor Grade of Clear Cell Renal Cell Carcinoma. AJR Am J Roentgenol (2014) 203:295-300. doi: 10.2214/AJR.13.12297

28. Pichler M, Hutterer GC, Chromecki TF, Jesche J, Kampel-Kettner K, Rehak P, et al. Histologic Tumor Necrosis is an Independent Prognostic Indicator for Clear Cell and Papillary Renal Cell Carcinoma. Am J Clin Pathol (2012) 137:283-9. doi: 10.1309/AJCPLBK9L9KDYQZP

29. Feng Z, Shen Q, Li Y, Hu Z. CT Texture Analysis: A Potential Tool for Predicting the Fuhrman Grade of Clear-Cell Renal Carcinoma. Cancer Imaging (2019) 19:6. doi: 10.1186/s40644-019-0195-7

Conflict of Interest: Author JR was employed by GE Healthcare company.

The remaining authors declare that the research was conducted in the absence of any commercial or financial relationships that could be construed as a potential conflict of interest.

Copyright ( 2021 Pei, Wang, Ren, Yin, Ma, Wang, Ma and Gao. This is an openaccess article distributed under the terms of the Creative Commons Attribution License (CC BY). The use, distribution or reproduction in other forums is permitted, provided the original author(s) and the copyright owner(s) are credited and that the original publication in this journal is cited, in accordance with accepted academic practice. No use, distribution or reproduction is permitted which does not comply with these terms. 